\title{
BMJ Open Injection Molded Autoclavable, Scalable, Conformable (iMASC) system for aerosol-based protection: a prospective single-arm feasibility study
}

\author{
James D Byrne, ${ }^{1,2}$ Adam J Wentworth, ${ }^{2,3}$ Peter R Chai, ${ }^{4}$ Hen-Wei Huang, ${ }^{2,3}$ \\ Sahab Babaee, ${ }^{3,5}$ Canchen Li, ${ }^{2}$ Sarah L Becker, ${ }^{3}$ Caitlynn Tov, ${ }^{3}$ Seokkee Min, ${ }^{2}$ \\ Giovanni Traverso (1) 3,5
}

To cite: Byrne JD,

Wentworth AJ, Chai PR, et al. Injection Molded Autoclavable, Scalable, Conformable (iMASC) system for aerosol-based protection: a prospective singlearm feasibility study. BMJ Open 2020;10:e039120. doi:10.1136/ bmjopen-2020-039120

- Prepublication history and additional material for this paper are available online. To view these files, please visit the journal online (http://dx.doi. org/10.1136/bmjopen-2020039120).

JDB, AJW, PRC and H-WH contributed equally.

Received 04 April 2020

Revised 12 June 2020

Accepted 15 June 2020

D) Check for updates

(c) Author(s) (or their employer(s)) 2020. Re-use permitted under CC BY-NC. No commercial re-use. See rights and permissions. Published by BMJ.

For numbered affiliations see end of article.

\section{Correspondence to}

Dr Giovanni Traverso; cgt20@mit.edu; ctraverso@ bwh.harvard.edu

\section{ABSTRACT}

Objective To develop and test a new reusable, sterilisable N95 filtering facepiece respirator (FFR)-comparable face mask, known as the Injection Molded Autoclavable, Scalable, Conformable (iMASC) system, given the dire need for personal protective equipment within healthcare settings during the COVID-19 pandemic.

Design Single-arm feasibility study.

Setting Emergency department and outpatient oncology clinic.

Participants Healthcare workers who have previously undergone N95 fit testing.

Interventions Fit testing of new iMASC system.

Primary and secondary outcome measures Primary outcome is success of fit testing using an Occupational Safety and Health Administration (OSHA)-approved testing method, and secondary outcomes are user experience with fit, breathability and filter replacement.

Results Twenty-four subjects were recruited to undergo fit testing, and the average age of subjects was 41 years (range of 21-65 years) with an average body mass index of $26.5 \mathrm{~kg} / \mathrm{m}^{2}$. The breakdown of participants by profession was $46 \%$ nurses ( $n=11), 21 \%$ attending physicians $(n=5)$, $21 \%$ resident physicians $(n=5)$ and $12 \%$ technicians $(n=3)$. Of these participants, four did not perform the fit testing due to the inability to detect saccharin solution on premask placement sensitivity test, lack of time and inability to place mask over hair. All participants $(n=20)$ who performed the fit test were successfully fitted for the iMASC system using an OSHA-approved testing method. User experience with the iMASC system, as evaluated using a Likert scale with a score of 1 indicating excellent and a score of 5 indicating very poor, demonstrated an average fit score of 1.75 , breathability of 1.6, and ease of replacing the filter on the mask was scored on average as 2.05.

Conclusions The iMASC system was shown to successfully fit multiple different face sizes and shapes using an OSHA-approved testing method. These data support further certification testing needed for use in the healthcare setting.

\section{INTRODUCTION}

Dwindling supplies of personal protective equipment (PPE) in hospitals is forcing
Strengths and limitations of this study

Development of a new N95-comparable mask that can be sterilised and reused.

- Mechanical testing of Injection Molded Autoclavable, Scalable, Conformable (iMASC) system determining stability under sterilisation conditions.

- Finite elemental analysis showcasing mask deformation and reaction forces from facial scans of 20 different wearers

- Testing of iMASC system among physicians, nurses and technicians with faces that were in different sizes and shapes.

- The iMASC system as a promising alternative sustainable solution to the dwindling supply of disposable N95 filtering facepiece respirators.

healthcare workers to reuse and clean PPE using anecdotal strategies, which may weaken the effectiveness of PPE in protecting workers from acquisition of COVID-19 disease. In some places, the complete lack of PPE has resulted in healthcare workers using PPE that may have variable droplet protection. ${ }^{1}$ Shortages of PPE have significant impact among healthcare workers who evaluate individuals with suspected and confirmed COVID-19 disease. ${ }^{12}$ First, individuals using PPE acquired outside of the hospital may inadvertently be using PPE without droplet protection resulting in inadequate protection. Second, workers without PPE will acquire infections, including COVID-19, at greater rates than those with adequate PPE. ${ }^{3}$ Infected healthcare workers may transmit disease to family members, worsening the pandemic. ${ }^{4}$ Third, with increased COVID-19 infection among healthcare workers, the available workforce to address sick patients decreases, resulting in increasing morbidity and mortality. ${ }^{4}$ There is, therefore, a critical 
need to develop innovative measures to generate safe, reusable $\mathrm{PPE} .^{5}$

Thus, we have designed and fabricated an Injection Molded Autoclavable, Scalable, Conformable (iMASC) system for aerosol-based protection with N95 material filters that can be inserted and replaced as needed. To understand the ability of our mask to conform to multiple face sizes and shapes, we have undertaken finite element (FE) analysis evaluating the deformability of the iMASC system. Lastly, we performed a prospective clinical trial for fit testing of our mask as well as qualitative assessment of the mask compared with the current N95 filtering facepiece respirators (FFRs). Our goal is to address the critical shortage of N95 FFRs to maximally protect healthcare workers and provide an enduring supply chain of N95 FFRs to reduce and prevent COVID-19 transmission among healthcare workers and patients.

\section{METHODS \\ Materials}

The mask material was DOW Corning QP1-250 Shore 50A liquid silicone rubber (LSR) sourced to Protolabs (Maple Plain, Minnesota, USA). The nasal bridge and elastic holders were $5 \mathrm{~mm}$ wide by $1 \mathrm{~mm}$ thick aluminium strips obtained from Amazon, and nylon elastic bands were obtained from a local fabric store. Adhesive for the nasal bridge was 3M Scotch-Weld (PR40). Filters were laser cut from 3M 1860 N95 FFRs. The filters were adhered to laser cut acrylic sheeting ( $3.2 \mathrm{~mm}$ thick, $46 \mathrm{~mm}$ diameter) (McMaster Carr, Product 8560K257) using fabric adhesive obtained from a local fabric store.

\section{iMASC fabrication}

Masks were designed in the three-dimensional (3D) computer-aided design (CAD) software SolidWorks (Dassault Systems) based on current 3M 1860 N95 FFRs that were in use at the hospitals in the Partners Healthcare network. Reusable face masks were then generated by Protolabs through injection moulding out of LSR. Elastic straps were used to secure the mask to the wearer's face. The mask used dual, replaceable filters. A $7.6 \mathrm{~cm}$ long aluminium strip was bonded across the bridge of the nose section of the mask similar to traditional N95 FFRs.

\section{Material testing}

To evaluate sterilisation of the iMASC system, the masks ( $\mathrm{n}=4$ per group) were exposed to a variety of sterilisation methods, including 10 cycles of autoclaving (dry cycle$121^{\circ} \mathrm{C}$ for $15 \mathrm{~min}$ ), $10 \mathrm{~min}$ soak in $1: 10$ bleach solution and 10 min soak in $100 \%$ isopropanol. These sterilisation methods were performed mutually exclusively. These solutions were selected to simulate on shift sterilisation by healthcare workers using standard hospital cleaning solutions. Mechanical testing according to ASTM D412 (Standard Test Methods for Vulcanised Rubber and Thermoplastic Elastomers) was performed on samples cut directly from the sterilised masks. Unpaired t-test was performed on tensile stress at maximum force between groups to evaluate for statistical differences.

\section{Face scans}

To obtain the 3D face geometry of the participants, we developed an IOS application (app) using the TrueDepth camera from an iPhone 11 to capture the face image of the participants. The app employs the ARKit developed by Apple for the use of face tracking in augmented reality to transform a two-dimensional image with depth information into a 3D mesh. The output 3D mesh would then be converted into a solid model for FE analysis.

\section{Deformation studies}

The commercial FE package ABAQUS/standard 2017 was used for simulating the deformation of the iMASC system. The 3D FE models were constructed by importing the CAD model of the mask from SolidWorks and scanned images of the participant faces. In all the analyses, we discretised the mask using four-node 3D linear tetrahedron elements with hybrid formulation (C3D4H Abaqus element type). The material behaviour of the elastomeric mask was captured using an almost incompressible Neo-Hookean hyperelastic model with Poisson's ratio of $v_{-} 0=0.499$ and density of $1.12 \mathrm{E} 3 \mathrm{~kg} / \mathrm{m}^{3}$ with directly imported stress-strain curves from mechanical testing. A simplified contact law ('surface-to-surface' type interaction) was assigned to the model with a penalty friction coefficient 0.2 for tangential behaviour and a 'hard' contact for normal behaviour. The top-middle edge of the mask was positioned to the node at the centre of the line connecting the eyes. The 'Quasi-static' dynamic implicit solver (*DYNAMIC module in Abaqus) was used. The mask was deformed by applying tensile forces along bands, as shown in online supplementary figure S1 using SMOOTH step amplitude curve, while completely constraining the motion of the face. The reaction force of the mask against the face as well as contact pressures were recorded as a function of applied load. Multiple levels of the reaction forces were exerted from the mask to the face, including $\mathrm{F}=0$ (undeformed), 4.5 (initial contact) and 10 (full contact) $\mathrm{N}$.

\section{Clinical studies}

Subjects were composed of adult Partners Healthcare staff including physicians, residents, nurses and technicians who were recruited on a voluntary basis and had undergone Occupational Safety and Health Administration (OSHA)-approved fit testing over the past year. Healthcare workers with facial hair were excluded from enrolment. Subjects were enrolled by study staff and gave informed verbal consent to participate in the study. Verbal informed consent was obtained due to noninvasive nature and short duration of the study. Following enrolment and consent, all subjects were briefed on the study procedure by the same member of the research team and then completed a baseline assessment to obtain 


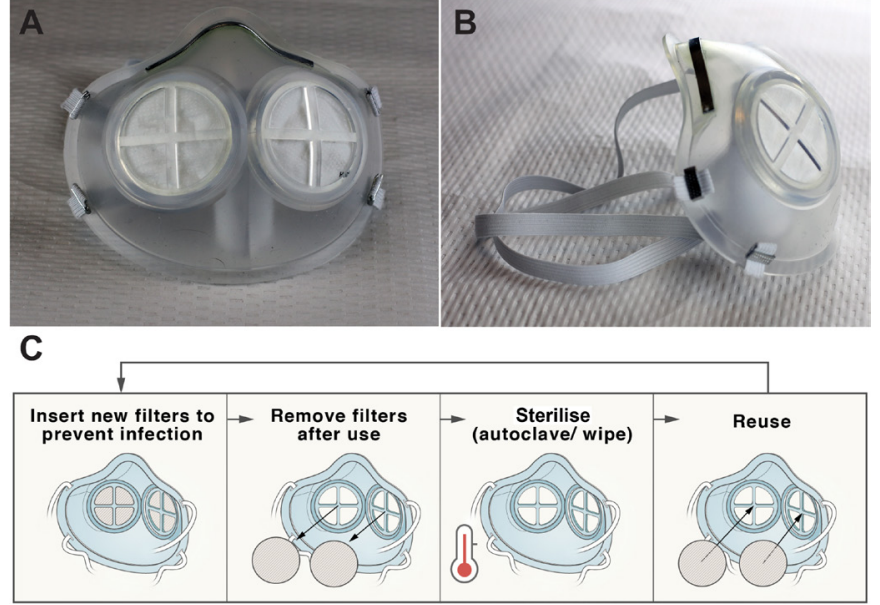

Figure 1 Injection Molded Autoclavable, Scalable, Conformable (iMASC) system for aerosol-based protection. (A) Front and (B) side images of the iMASC system. (C) Workflow for sterilisation and reuse of iMASC system.

general demographic information and ensure they had previously been fit tested successfully.

Subjects underwent fit testing in accordance to the Saccharin Solution Aerosol Protocol per OSHA $\$ 1910.134$ using the Gerson Respirator Fit Test kit (Gerson part no: 065000 , Middleboro, Massachusetts, USA) with the saccharin solution. The fit testing was performed by a member of the study staff. This fit test system was the same system used for fit testing healthcare workers at the hospitals in the Partners Healthcare system. After successful completion of the threshold screening test, subjects donned the iMASC system and a hood with a fitted collar. They were instructed to report if they could taste the test solution. A nebuliser of the saccharin solution was inserted into the hole in the front of the hood and sprayed at the same concentration (10, 20 or 30 squeezes) as the subject was able to taste in their initial threshold test. The subject was instructed to perform the following exercises while the aerosolised solution was replenished every 30 s: normal breathing, deep breathing, turning the head side to side, moving the head up and down, counting backwards from 100, grimacing, bending over and finally normal breathing for a second time. If the subject at any time during the fit test was able to taste the solution, they indicated to the study staff and the test was considered failed. If the subject did not report tasting the solution the test was considered passed. Subjects who passed the fit test were introduced to how to properly replace the filter with a demonstration by study staff. Subjects were then asked to replace the filter and perform a user seal check to ensure an adequate fit. This procedure allowed us to simulate the replacement of filters by healthcare workers prior to the start of a workday. Finally, subjects completed an exit assessment where they ranked fit, breathability and difficulty of replacing the filter according to a Likert scale. Subjects were also asked about their willingness to wear the mask compared with either a surgical mask or an N95 mask. All testing was performed at Brigham and Women's Hospital.

\section{RESULTS}

\section{Design and generation of injection-moulded LSR mask}

The iMASC system was designed to function as an N95 FFR-comparable face mask (figure 1). The shape of the iMASC system was modelled from disposable regular N95 FFRs used in the hospital. Medical grade LSR was identified as an optimal material for mask fabrication due to its conformable capacity, sterilisability through multiple methods and compatibility with injection moulding for fabrication scalability. The weight of the iMASC system was $44.84 \pm 0.05 \mathrm{~g}(\mathrm{n}=3)$ compared with $10.41 \pm 0.13 \mathrm{~g}(\mathrm{n}=3)$ of current N95 FFRs. We employed a dual-filter approach similar to half-mask elastomeric respirators to increase breathability and filtration area. ${ }^{5}$ A single regular N95 FFR generated up to five filters for the iMASC system, thus extending the N95 material use.

\section{Characterisation of mask material after sterilisation}

An advantage of the iMASC system over the half-mask respirators is the methods of sterilisation (see online supplementary table $\mathrm{S} 1$ ). We have performed tensile tests of the mask material after 10 autoclave cycles and 5 min in a 1:10 bleach solution and 70\% isopropyl alcohol. We found that 10 autoclave cycles make the mask slightly stiffer, while the bleach soak resulted in no change and the isopropanol alcohol soak makes the material less stiff (online supplementary figure S2). Evaluation of the tensile stress at maximum forces between groups was found to not be significantly different $(p>0.05)$. Despite these small changes in tensile strength, there were no gross differences in the mask compared with the nonsterilised mask.

\section{FE analysis for mask deformation on different face shapes and sizes}

We used non-linear FE analyses (see 'Deformation studies' in Methods) to evaluate the deformation of the flexible mask frames while wearing and determine the forces required to keep the mask in place across a range of subject faces. In figure 2A, we reported the numerical snapshots of the face mask when subjected to the strap's tensile loads, denoted by $T$ shown in online supplementary figure S1, and monitored the deformation of the mask at different levels of the reaction force exerted from the mask to the face. The colour maps represent the distribution of displacement's magnitude, $U$, showing relatively large deformation of the mask required to fit in to the subject face. We also calculated the normal contact forces, $F^{N}$, and contact pressures, $P$, as a function of $F$ to evaluate the interaction between the mask and face. In figure $2 \mathrm{~B}$, the distribution of the $F^{N}$ are shown at the different $F$. As expected, no $F^{N}$ was recorded at $F=0$ $\mathrm{N}$. By pulling the straps, the mask starts to be engaged with the face, and at $F=4.5 \mathrm{~N}$ the maximum $F^{N}$ occurs 

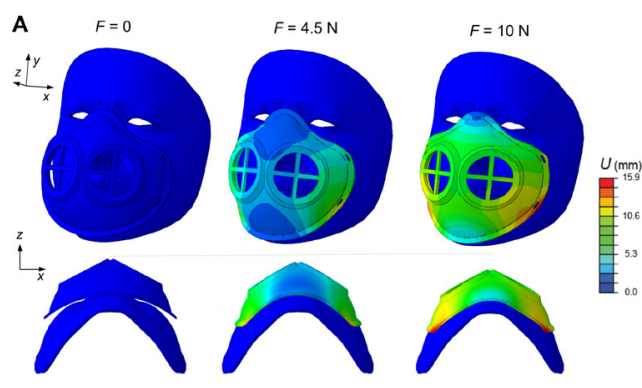

C
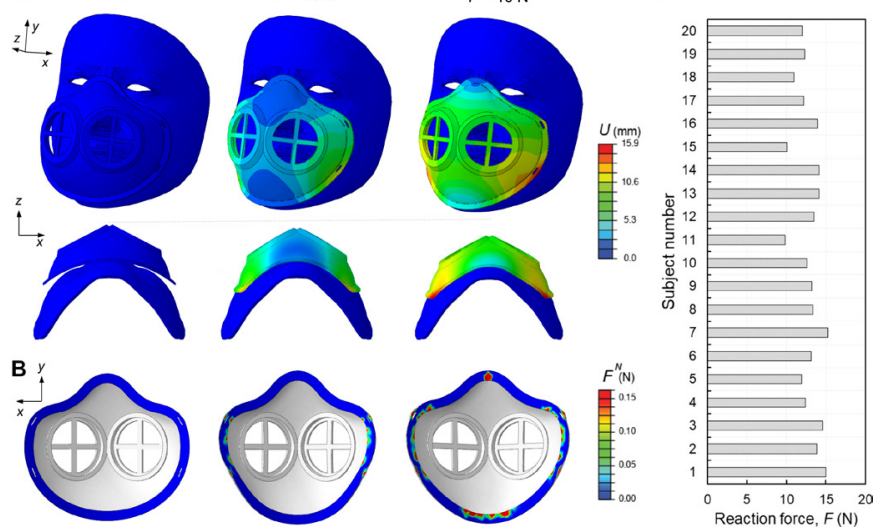

Figure 2 Finite element modelling of flexible masks. (A) Representative numerical images showing the deformation of the elastomeric mask at different levels of reaction forces, $\mathrm{F}=0,4.5$ and $10 \mathrm{~N}$ in two different views (top and bottom rows). The colours represent the magnitude of displacement field, U. (B) The corresponding distribution of the normal contact forces, $\mathrm{F}^{\mathrm{N}}$, between the mask and face. (C) Reaction forces for the subject numbers $n=1,2,3 \ldots, 20$ computed from simulations.

around the cheek. Further pulling the straps $(F=10 \mathrm{~N})$ induces a relatively higher $F^{N}$ along the edge of the mask in the cheek and chin (lower lips) rather than the nose and cheekbones. This is a signature of the need to the aluminium strip to bond across the bridge of the nose to enhance the contact pressure.

Next, we estimated the reaction force required to achieve an average contact pressure of $P=10 \mathrm{KPa}$ (relatively uniformly distributed along the edge of the mask) as a higher limit of the contact pressure that results in a suitable fit between the mask and skin faces. ${ }^{6}$ This reaction force is equivalent to the force applied through the straps. In figure 2C, we reported the reaction forces for

A
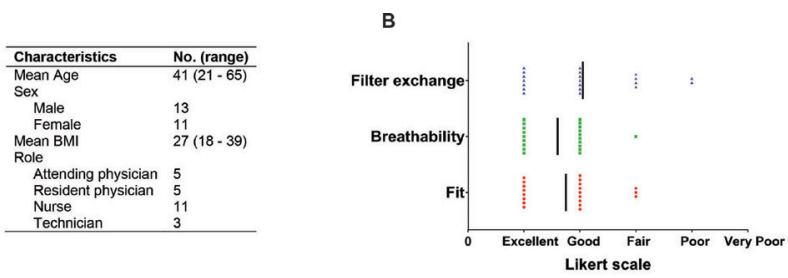

C

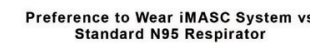

D
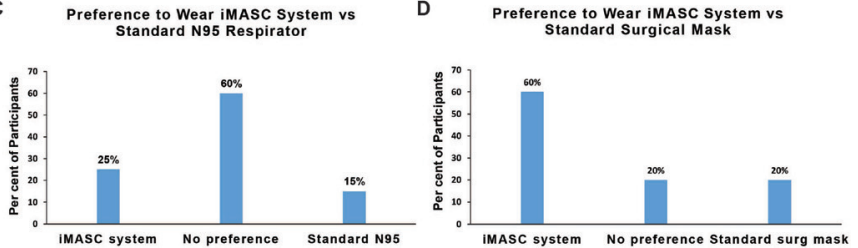

Figure 3 Fit testing of Injection Molded Autoclavable, Scalable, Conformable (iMASC) system in healthcare workers and their user experience. (A) Demographics of participants $(n=24)$ enrolled in fit testing clinical trial. (B) User experience $(n=20)$ with the mask based on a Likert scale. User preferences $(n=20)$ comparing the iMASC system to the (C) standard surgical mask and (D) N95 respirators. BMI, body mass index.
20 different subjects, ranging from 9.5 to $15 \mathrm{~N}$. These variations are due to the difference in shape and size of the subject's faces especially in the jaw and cheekbone parts. Through application of these forces via the straps combined with the aluminium strip across the nose bridge, the mask should remain in place.

\section{Clinical trial evaluating mask fitting}

In a prospective trial, we enrolled 24 healthcare workers at a large, urban, academic medical centre who had been previously certified to wear a N95 respirator into our IRBapproved study. We excluded individuals with facial hair or those who had failed an N95 fit test. Consenting individuals were subject to a fit test as defined by OSHA. ${ }^{78}$ Figure $3 \mathrm{~A}$ shows the demographics of the participants, and online supplementary figures S3 and S4 showcase the 3D facial reconstructions demonstrating variability of facial sizes and shapes among the participants. The average age of participants was 41 years with a range of 21-65 years with an average body mass index of $26.5 \mathrm{~kg} /$ $\mathrm{m}^{2}$. The breakdown of participants by profession was $46 \%$ nurses $(n=11), 21 \%$ attending physicians $(n=5), 21 \%$ resident physicians $(n=5)$ and $12 \%$ technicians $(n=3)$. Of these participants, four did not perform the fit testing (one due to inability to detect saccharin solution on premask placement sensitivity test, two due to time and one due to the inability to get the elastic straps over her hair and face).

All participants $(n=20)$ who performed the fit test successfully completed the fit test as part of the hospital annual policy. All participants passed their fit test and were also able to successfully replace the filter into the mask, resulting in a $100 \%$ success rate for both fit testing and filter exchange. User experience with the iMASC system was evaluated using a Likert scale with a score of 1 indicating excellent and a score of 5 indicating very poor. Participants scored the fit of the iMASC system as excellent (eight participants), good (nine participants) or fair (three participants) (figure 3B). Participants scored the breathability of the iMASC system as excellent (9 participants), good (10 participants) or fair (1 participants). Finally, participants scored the filter replacement of the iMASC system as excellent (seven participants), good (seven participants), fair (four participants) or poor (two participants). Participants' preference to wear the iMASC over a surgical mask or an N95 respirator was also assessed. Sixty per cent of participants indicated they would be willing to wear our mask instead of a surgical mask, with $20 \%$ indicating no preference between our mask and a standard surgical mask and $20 \%$ indicating they would prefer to wear a surgical mask (figure 3C). When asked about preference to wear our mask instead of an N95 FFR, 25\% of participants indicated they would prefer to wear our mask and $60 \%$ indicated no preference between our mask and a N95 FFR, with only $15 \%$ indicating they would prefer to wear a standard issue N95 FFR (figure 3D). 


\section{DISCUSSION}

During times of pandemics, it is essential to protect healthcare workers from infection and transmission of disease with adequate PPE. ${ }^{49}$ As stocks of N95 FFRs have reduced, healthcare workers are forced to find alternative strategies of protection, including resterilising masks and using alternative mask materials that may result in less protection. ${ }^{9}{ }^{10}$ Our approach here was to develop a scalable, reusable face mask that can extend the amount of N95 material. The iMASC system withstood decontamination using three methods and was shown to successfully fit multiple different face sizes and shapes using an OSHAapproved testing method. The iMASC system could be scaled up for use across many locations once additional certification testing, including the sodium chloride aerosol challenge test, dioctyl phthalate aerosol test, and inhalation and exhalation tests, has been completed. By selecting injection moulding as the fabrication technique for the iMASC system, we believe we possess a fundamental advantage to other initiatives using 3D printing techniques because injection moulding is highly scalable and has decreased production time when compared with 3D printing.

These are initial proof-of-concept studies and have some limitations. First, the small sample size and single institutional nature of this prospective study limit generalisability and warrants evaluation in a larger cohort involving multiple institutions. As a result of the lack of availability of standard N95 FFRs, the iMASC system was not compared with standard-of-care N95 FFRs. Previous studies have demonstrated that a respirator user gains experience with subsequent donnings and may result in improved fit-test pass rate biasing our results ${ }^{11-13}$; thus, it will important to assess fit testing in inexperienced subjects. While Bitrex is the preferred choice for fit test solution as a leak detection, ${ }^{14}$ saccharin was chosen due to availability and use in OSHA-approved qualitative fit tests. Additional development for smaller face sizes and shapes is warranted since the iMASC system was modelled from the 3M 1860 model. Furthermore, all testing was performed in North America, and it is possible face shapes and sizes may differ for workers outside of this region. Modifications to the filter system and elastic straps would likely improve the fit and robustness of the mask. All post-injection moulding manufacturing steps were completed in-house and in large scale production would be outsourced to contracted manufacturers with greater quality control of filter components. Further, the testing of mechanical properties after combinations of different sterilisation techniques could provide a better representation of what would be used in the hospital. Additional quantitative fit testing, extended wearer testing and certification testing, including NIOSH 42 CFR part 84 (or equivalent), will be needed to validate the iMASC system for use in the healthcare setting as qualitative fit testing is unable to verify the protection factor of the respirator. To source additional filter materials in the future, we will plan to perform filter efficiency testing on these materials, such as the NIOSH Standard Test Procedure (STP) TEB-APR-STP-0059.

Newer face masks, such as our iMASC system, have potential to resupply and sustain hospitals with effective N95-comparable masks. Furthermore, a 2018 consensus report from the National Academies of Engineering, Science, and Medicine recommended that the durability and reusability of elastomeric respirators made them desirable for stockpiling for emergencies. ${ }^{5}$ This approach could be applicable to users outside of the healthcare setting, including people in the research, home improvement and manufacturing settings.

\section{Author affiliations}

${ }^{1}$ Harvard Radiation Oncology Program, Brigham and Women's Hospital, Boston, Massachusetts, USA

${ }^{2}$ David H. Koch Institute for Integrative Cancer Research, Massachusetts Institute of Technology, Cambridge, Massachusetts, USA

${ }^{3}$ Division of Gastroenterology, Brigham and Women's Hospital, Boston,

Massachusetts, USA

${ }^{4}$ Department of Emergency Medicine, Brigham and Women's Hospital, Boston, Massachusetts, USA

${ }^{5}$ Department of Mechanical Engineering, Massachusetts Institute of Technology, Cambridge, Massachusetts, USA

\section{Twitter Giovanni Traverso @cgtraverso}

Acknowledgements We thank Ania Hupalowska for her illustrations of the clinical workflow. We also thank Professor R Langer for helpful discussions around mask development.

Contributors JDB and AJW designed and fabricated the iMASC system, assisted with the clinical trial, analysed and interpreted data, and wrote the manuscript. PRC performed the clinical trial, analysed and interpreted data, and wrote the manuscript. $\mathrm{H}-\mathrm{WH}$ and $\mathrm{SB}$ designed the face scanning and performed FE analysis modelling, analysed data and wrote the manuscript. SB, CT and SM analysed data and designed prototypes. GT supervised, reviewed the data and edited the manuscript.

Funding This work was supported in part by the Prostate Cancer Foundation. JDB was supported by the Prostate Cancer Foundation Young Investigator Award. GT was supported in part by the Department of Mechanical Engineering, MIT, and Brigham and Women's Hospital. PRC was supported by NIHK23DA044874, and investigator-initiated research grants from e-ink, Gilead Sciences, Philips Biosensing and the Hans and Mavis Lopater Psychosocial Foundation. Support for the materials and supplies was from discretionary funds to GT from Brigham and Women's Hospital and the Department of Mechanical Engineering, MIT.

Competing interests None declared.

Patient and public involvement Patients and/or the public were not involved in the design, conduct, reporting or dissemination plans of this research.

Patient consent for publication Not required.

Ethics approval Partners Healthcare Institutional Review Board (IRB) approval was obtained prior to any human testing of the iMASC system (Partners IRB 2020P000852).

Provenance and peer review Not commissioned; externally peer reviewed.

Data availability statement Data are available in a public, open access repository. The authors declare that the data supporting the findings of this study are available within the paper and its supplementary information files.

Open access This is an open access article distributed in accordance with the Creative Commons Attribution Non Commercial (CC BY-NC 4.0) license, which permits others to distribute, remix, adapt, build upon this work non-commercially, and license their derivative works on different terms, provided the original work is properly cited, appropriate credit is given, any changes made indicated, and the use is non-commercial. See: http://creativecommons.org/licenses/by-nc/4.0/.

\section{ORCID iD}

Giovanni Traverso http://orcid.org/0000-0001-7851-4077 


\section{REFERENCES}

1 Ranney ML, Griffeth V, Jha AK. Critical supply shortages - the need for ventilators and personal protective equipment during the Covid-19 pandemic. N Engl J Med 2020;382:e41.

2 Livingston E, Desai A, Berkwits M. Sourcing personal protective equipment during the COVID-19 pandemic. JAMA 2020.

doi:10.1001/jama.2020.5317. [Epub ahead of print: 28 Mar 2020].

3 Adams JG, Walls RM. Supporting the health care workforce during the COVID-19 global epidemic. JAMA 2020. doi:10.1001/ jama.2020.3972. [Epub ahead of print: 12 Mar 2020].

4 The Lancet. COVID-19: protecting health-care workers. Lancet 2020;395:922.

5 National Academies of Sciences, Engineering, and Medicine, Health and Medicine Division, Board on Health Sciences Policy, Committee on the Use of Elastomeric Respirators in Health Care. Reusable elastomeric respirators in health care: considerations for routine and surge use. Washington (DC): National Academies Press (US), 2018

6 Brill A-K, Pickersgill R, Moghal M, et al. Mask pressure effects on the nasal bridge during short-term noninvasive ventilation. ERJ Open Res 2018;4:00168-2017-2017.

7 Occupational Safety and Health Standards. OSHA 1910.134 appendix A: fit testing procedures (mandatory), 2017.
8 U.S. Department of Labor. Temporary enforcement guidance healthcare respiratory protection annual fit-testing for N95 filtering Facepieces during the COVID-19 outbreak, 2020.

9 Feng S, Shen C, Xia N, et al. Rational use of face masks in the COVID-19 pandemic. Lancet Respir Med 2020;8:434-6.

10 Maclntyre CR, Seale H, Dung TC, et al. A cluster randomised trial of cloth masks compared with medical masks in healthcare workers. BMJ Open 2015;5:e006577.

11 Or P, Chung J, Wong T. A novel approach to fit testing the N95 respirator in real time in a clinical setting. Int $J$ Nurs Pract 2016;22:22-30.

12 Lee MC, Takaya S, Long R, et al. Respirator-fit testing: does it ensure the protection of healthcare workers against respirable particles carrying pathogens? Infect Control Hosp Epidemiol 2008;29:1149-56.

13 Hannum D, Cycan K, Jones L, et al. The effect of respirator training on the ability of healthcare workers to pass a qualitative fit test. Infect Control Hosp Epidemiol 1996;17:636-40.

14 McKay RT, Davies E. Capability of respirator wearers to detect aerosolized qualitative fit test agents (sweetener and Bitrex) with known fixed leaks. Appl Occup Environ Hyg 2000;15:479-84. 